\title{
Substantiation of the technological scheme of pneumatic grain seeder of subsurface dense sowing
}

\author{
Azamat E. Bogus ${ }^{1, *}$, and Arsenyi D. Kuzmenko ${ }^{1}$ \\ ${ }^{1}$ FSBEIHE Kuban State Agrarian University named after I.T.Trubilin, Krasnodar, Russia
}

\begin{abstract}
The article describes the shortcomings of existing structures and units of pneumatic grain seeders. There was analyzed the technological process of distribution of seed material in distribution systems. There were described the various options of developed designs of dosing and distributive systems allowing to increase the sowing machines efficiency. To improve the efficiency of sowing machines, it is proposed to establish a distribution of seeds with a downward flow, which will increase the uniformity of seed supply to coulters and, consequently, increase the uniformity of their distribution over the field surface.
\end{abstract}

Relevance. Getting high results is an urgent task of agricultural production. As with seeding the foundations of future crops, the uniformity of plant distribution on the area of nutrition is important for the future yields. The most efficient area of plant nutrition is obtained under subsoil scattered method of sowing, because only in this way such area is approaching the best one, i.e. round.

One of the significant shortcomings of existing pneumatic seeders with a central dosing system is a limited seeding rate. An increase in the seeding rate of more than $190 \mathrm{~kg} / \mathrm{ha}$ leads to clogging of pneumatic shanks [1].

These disadvantages are due to the following. The central dosing system of the wellknown pneumatic seeder, which implements the principle of central dosing of seeds with their pneumatic transportation into coulters, is a two-stage system.

In addition, the presence of two stages of seed distribution reduces the throughput ability of the central dosing system of the seeder. It limits the increase of advancing speed of the seeder in the process of sowing and as a result of its productivity.

We use two fans to reduce the non-uniformity of seed distribution between coulters up to $5 . .7 \%$ in the two-stage design of the central dosing system of the seeder. The presence of a second fan and a distribution head on the vertical column of the first stage of the seed distributor increases the materials consumption of the seeder, complicates its design and as a result reduces the reliability and increases the complexity of maintenance $[2,3]$.

\footnotetext{
* Corresponding author: azamat089@gmail.com
} 
The process of the seed flow separation in dividing heads of the seeder is carried out by impacting the seeds on the surface and their reflecting from it. Upon impact, the seeds undergo compression deformation. The upper limit of the permissible speed of air movement during pneumatic seed transportation in the vertical column of the seed distributor of the seeder is limited by the risk of seed injury and it should not exceed 27 $\mathrm{m} / \mathrm{s}$ according to the recommendation. It limits the throughput capacity of the central dosing system of the seeder and as a result the productivity of its operation during sowing.

Limitation of aerodynamic characteristics (speed of air flow, its dynamic force, air flow) of the central dosing system of the seeder, which appears due to the presence of two stages of seed distribution and the use of impact as the main factor in the separation of seeds in the dividing heads, creates the potential possibility to clog the diverting pneumatic hoses with seeds. The clogging of diverting pneumatic hoses increases the complexity of seeders maintenance in the process of sowing.

Seeds remain and accumulate at the bottom of the chamber of dividing heads of seed distributors in the result of changes in the aerodynamic characteristics of air flow during the sowing process. The need to remove them from the chambers of the dividing heads, in particular when changing one variety or a cultivar for other, increases the laborintensiveness of the seeder maintenance and the time spent on its downtime [4, 5].

We have a pneumatic dispenser for seeds of the seeder with a central dosing system containing a dividing head connected with the diverting pneumatic hoses with furrowers and located in the vertical column, which is connected with the pneumatic lines, in the form of a Venturi pipe connected with an air chamber, dividing air flows and pneumatically connecting the blast engines, two tankers with the measuring systems-hoppers, connected to Venturi tubes via the distribution valves.

The considerable non-uniformity in seed distribution between furrowers and low throughput ability of a pneumatic seed dispenser are the disadvantages of the known pneumatic seed dispenser.

These disadvantages are due to the following. In the known pneumatic seed dispenser, the seed flow is divided into a single-stage scheme in the dividing heads of a round shape, i.e. in the form of a body of rotation having a cover and a bottom. And the diameter of the dividing head is greater than its height. This ratio of these dimensions is performed in order to bring the surface of the cover to the inlet of the vertical column located in the bottom of the chamber. This provides a more intense impact of seeds on the lid. The air flow transporting seeds enters the dividing head through the holes in the bottom, and seeds are hit about the lid and reflecting, they are distributed in diverting pneumatic hoses. The seed non-uniformity between furrow-makers-coulters reaches $9 \ldots 16 \%$. Significant deviations from the given seeding rate, allowed by furrowers-coulters are the consequence of such a significant non-uniform distribution of seeds between furrowers-coulters. It reduces the uniformity of seed distribution on the field surface and as a result - the crop productivity of cultivated crops [6].

The seeds in the process of splitting on diverting pneumatic hoses are hit on the lid of the dividing head and are subjected to dynamic deformation of pressing. The upper limit of the permissible speed of air movement during pneumatic transportation of seeds is limited by the risk of seed damage and it should not exceed $27 \mathrm{~m} / \mathrm{s}$ according to the recommendation.

This limits the throughput capacity of the pneumatic seed distributor and as a result - an increase in the advancing speed of the sowing unit during operation, reducing the productivity of its work.

In addition, the design of the bottom of the inner part of the dividing head has horizontally located areas where seeds are delayed and accumulated. When changing the seeding of one variety or a cultivar to another, it is required to remove these seeds from the 
dividing head, which increases the complexity of the seeder maintenance during its operation.

The closest in technical essence and the achieved result to the proposed device is a pneumatic seeder with a central dosing system containing sequentially connected sources of positive air pressure by a pneumatic conduit, the hopper with feeder reel-to-reel type and seed distributor, with one division stage of seeds transported by the air flow and a dividing head having a chamber with a lateral surface in the form of a body of rotation, lid and bottom, and mounted on a vertical column equipped with a turbulator made in the form of corrugations or rings, fixed to the inner surface of the vertical column. And the round dividing head of the seed distributor is connected with outlet seed hoses with coulters.

The disadvantages of the device, taken as a prototype, are a significant uneven seed distribution between the seeders and as a consequence, a high uneven seed distribution in a furrow with an ordinary method of sowing and on the surface of the field with scattered subsurface sowing, as well as a low throughput ability of the central dosing system, a significant labor-intensiveness of the seeder maintenance during operation, the complexity of the design and materials consumption [7].

These disadvantages are due to the following. Design of a round head seed dispenser allows the uneven seed distribution in coulters which is equal 9 and $16 \%$, which reduces the uniformity of the distribution of seeds on the surface of the field and ultimately - the crop of cultivated crops.

In the process of seed distribution along the outlet pneumatic hoses, the seeds are reflected from the surface of the cover of the dividing head, which may be flat, concave or convex. Seeds that are hit on the surface of the lid are subjected to dynamic compression deformation. The upper limit of the permissible speed of air movement during pneumatic seed transportation is limited by the risk of seed damage and it should not exceed $27 \mathrm{~m} / \mathrm{s}$ according to the recommendation. This limits the increase of the advancing speed of the sowing unit during the sowing process and, as a consequence, the productivity of its work [8].

The presence of a turbulator in a vertical column complicates the design of the known pneumatic seeder. In addition, the design of the chamber of the dividing head has horizontally arranged areas in which the seeds are delayed and accumulated during sowing. When moving to the sowing of another variety or culture, it requires the removal of these seeds, which increases the complexity of the seeder maintenance.

The technical solution of the problem is to increase the uniformity of seed distribution between the seeder coulters. The task is achieved in that in the pneumatic seeder with a central dosing system containing the source of excess air pressure connected by a pneumatic line, the hopper with reel-to-reel type feeder and distributor of seeds, which has a chamber, the lateral surface of which is made in the form of a body of rotation having a lid and a bottom connected to outlet pneumatic hoses with coulters according to the invention, the bottom of the chamber of the seed distributor is convex and nipples are placed to its perimeter, at the same time, the final section of the pneumatic pipeline is divided into at least two pneumatic channels connected to the lateral surface of the seed distributor chamber above the nozzles of the diverting pneumatic hoses in which the through holes are made [9].

In the preferred type of a pneumatic seeder with a central dosing system, the top of the convex bottom of the seed distributor chamber is located at the level of the connection of the pneumatic channels to the lateral surface of the seed distributor chamber, and the surface of the convex bottom is provided with ribs mounted on its generator and between the nozzles of the diverting pneumatic hoses. 
In other preferred variant of a pneumatic seeder with a central dosing system, the connections of the pneumatic channels with the chamber of the seed distributor are arranged against each other.

The pneumatic seeder with a central dosing system having through holes of diverting pneumatic hoses which are located along their longitudinal axes with the possibility of individual overlap in the following type.

The inner surface of the pneumatic pipeline section located in front of the divider of the pneumatic flow is provided with lugs in other type of a pneumatic seeder with a central dosing system.

The novelty of the claimed technical solution lies in the fact that due to the convex bottom of the chamber of the seed distributor, the formation of an air flow entering the nozzles of the diverting pneumatic hoses and at the same time the supply of seeds from the upper part of the seed distributor in the pipe of the diverting pneumatic hoses is provided, i.e. the separation of the seed flow in the descending air flow and the movement of seeds under its own weight is used. Besides it, the parts which could accumulate seeds in the process of operation are eliminated, i.e. the self-cleaning of the chamber from seeds is provided, preventing variety mixing.

The location of the nozzles for the diverting pneumatic hoses along the perimeter of the bottom also ensures the separation of the seed flow in the descending air flow.

The division of the final section of the pneumatic pipeline into at least two pneumatic channels and their connection to the lateral surface of the seed distributor chamber over the nozzles of the diverting pneumatic hoses ensures the formation of the fluidized state of seeds in the upper part of the chamber due to the interaction of advancing air flows coming out of pneumatic channels, passing from pneumatic channels, coming in the upper part of the chamber into a state of intense turbulent air, together with the seeds moving in it. It provides the increase of uniformity of seed distribution along the upper part of the chamber and as a consequence, the uniformity of their distribution falling down and further along nozzles of pneumatic doses.

The connection to the lateral surface of the seed distributor chamber above the nozzles of pneumatic hoses ensures the separation of seed flow in descending air flow.

The through holes in pneumatic hoses allows to regulate the air flow in these hoses, preventing their clogging with seeds and ensuring a stable flow of the technological process.

The location of the top of the convex bottom of the seed distributor chamber at the level of the connection of pneumatic channels to the lateral surface of the seed distributor chamber increases the degree of influence of the bottom on the separation of the seed flow in the descending air flow.

The location of the connection between pneumatic channels and the distributor chamber opposite each other increases the turbulent state of the medium and, as a consequence, the intensity of the fluidized state of the seeds in the upper part of the chamber, which means their uniformity of dispersion in the upper part of the chamber.

The location of the through holes of the diverting pneumatic hoses along their longitudinal axes with the possibility of individual overlap provides a wide range of adjustment of aerodynamic characteristics of the air flow in diverting pneumatic hoses.

The supply of lugs of the inner surface of the section of the pneumatic pipeline, located in front of the divider of the pneumatic flow creates a turbulent state of the air flow in this section of the pneumatic pipeline, providing intensive mixing of seeds in the cross section of the pneumatic pipeline, which ultimately contributes to an increase in the uniformity of seed distribution to the diverting pneumatic hoses and it means along the coulters of seeders. 
The analysis of the properties of features of the claimed device and the properties of features of the detected prototype and analog showed that the features of the claimed device provides enhanced properties of the prototype - increases the uniformity of the seed distribution on the coulters and the throughput ability of the central dosing system of the pneumatic seeder.

Pneumatic seeder with central dosing system containing a frame 1, with supporting wheels 2 , which houses the engine 3 and connected in series with the pneumatic conduit 4 to a source of positive air pressure - fan 5, a central hopper 6 with a dispenser 7 of a reel-to-reel type, divider of pneumatic flow 8 and seed dispenser 9. Seed distributor 9 includes the chamber 10, lateral surface 11 , which is made in the form of a body of rotation and a blacket 12, which is mounted to the frame 1 (Fig. 1). The chamber 10 has the lid 13 and the bottom 14. Fastening of the blacket 12 can be to the lateral wall 11 of the chamber 10 or to the bottom 14. The bottom 14 is convex, for example, in the form of a cone, or in the form of cut off cone, in the form of a body of rotation with cycloids. The diameter of the chamber 10 of the seed distributor 9 is higher of its height. The preferable relation of the diameter of the height of the chamber is equal 1:1,5. Nipples 15 are located along the perimeters of the bottom 14, which are connected with diverting pneumatic hoses 16 with working bodies for planting of seeds into soil - coulters 17, made in the type of a lancet lug. The final part of the pneumatic cable 4 is divided by the divisor of the pneumatic cable 8 in two or four pneumatic channel 18, adjusted to the lateral surface 11 of the chamber 10 over nipples 14 of diverting pneumatic hoses 16 . Pneumatic channels 18 are connected in the upper part of the lateral surface 11 of the chamber 10 in their opposite sides and in the preferable type the connection of four pneumatic channels 18 are located opposite each other. The top of the convex bottom 14 is on the level of connection of the pneumatic channels 18 to the lateral surface 11 of the chamber 10 of the seed distributor 9 .

Pneumatic seeder with a central dosing system works as follows. When the seeder moves across the field, the seeds from the centralized hopper 6 , passing through the dispenser 7 , enter the air duct 4, are picked up in it by the flow of air created by the source of excess air pressure - fan 5. Passing through the pneumatic duct 4, located in front of the seed distributor 9 in a place where the inner surface of the site is provided with lugs, the seeds are mixed in a turbulent air flow, distributed more evenly in it. The pneumatic flow, transporting the seeds, enters through the divider of the pneumatic flow 8 and at least two air channels 11 into the chamber 10 of the seed distributor 9, losing the air flow speed in the chamber 10 , and the air flows separated by the pneumatic channels 18 , interacting, increase the turbulence in the upper part of the chamber 10, while the transported seeds here pass into a fluidized state, distributing and winding throughout the volume of the upper part of the chamber 10. Part of the seeds located in the upper part of the seed distributor chamber under the action of its own weight falls down and gets on the convex surface of the bottom 14 , then gets into the nozzles 15 of the diverting pneumatic hoses 16 . In this case, the ribs on the convex surface of the bottom 14 direct the movement of seeds in the direction of the holes of nozzles 15 and prevent their movement in the plane of the crosscut section of the chamber 10 .

The air flow creates excessive pressure in the chamber 10 of the seed distributor 9 , under the action of which, carrying seeds, randomly moving in the upper part of the chamber 10 and is pushed between the ribs 19 on the convex bottom surface 14, and escapes through the openings of the nozzles 15 and further via the outlet 16 in pneumatic hoses 11 and then into the soil. 


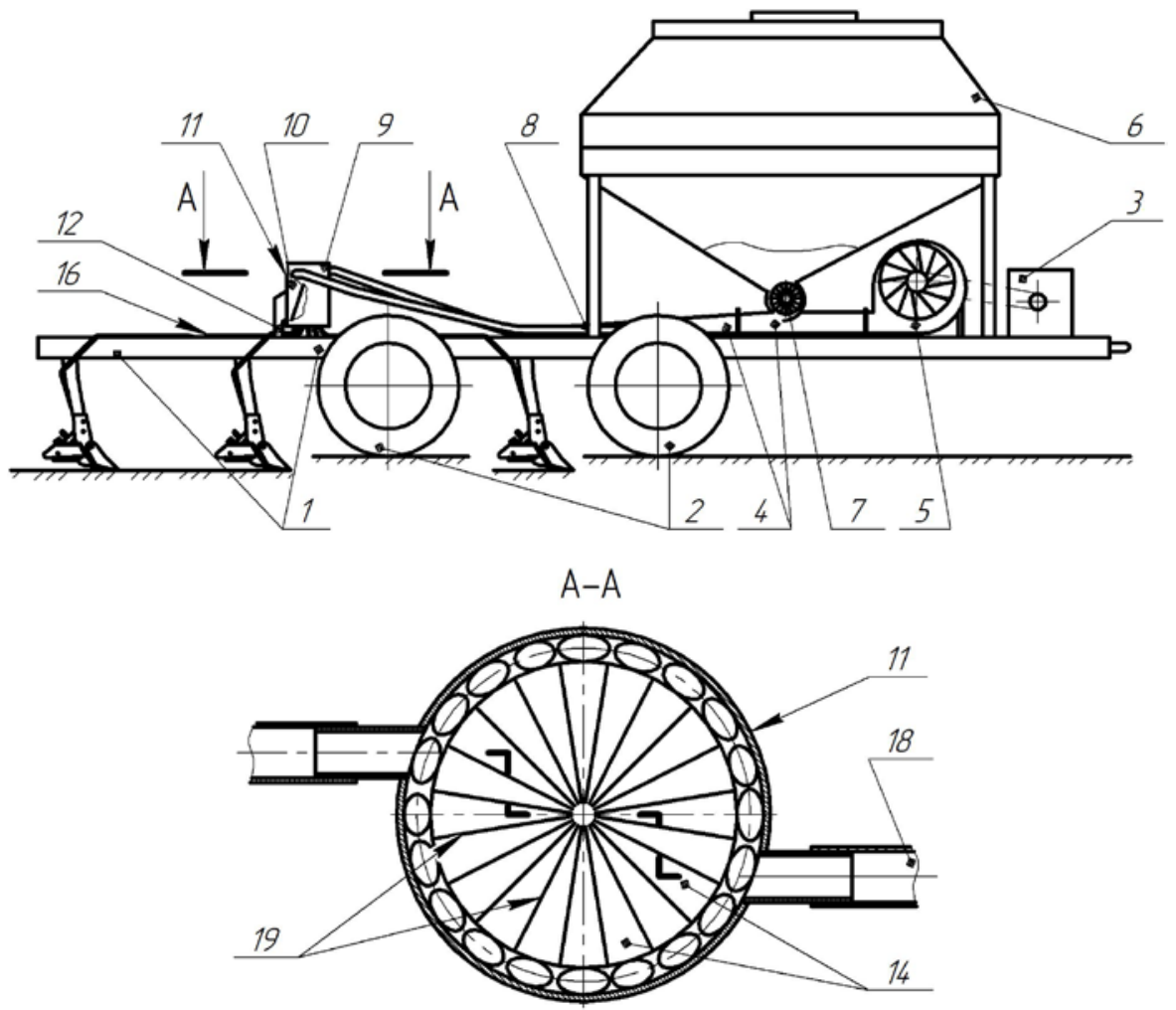

1 - frame; 2 - wheels; 3 - the engine; 4 - pneumatic conduit; 5 - fan; 6 - central hopper; 7 dispenser; 8 - pneumatic flow; 9 - seed dispenser; 10 - chamber; 11 - lateral surface; 12 - blacket; 13

- lid; 14 - bottom; 15 - nipples; 16 - pneumatic hoses; 17 - coulters; 18 - pneumatic cable.

Fig. 1. Pneumatic seeder with a central dosing system

At the location of the connection of the pneumatic ducts 4 with the chamber 10 against each other, the air flows coming out of them mixing, extinguish their kinetic energy and the seeds they transport. When using in the design of the seed distributor more than two pneumatic ducts 18 connected to one chamber 10, the turbulent state increases interacting air flows and random movement of seeds in the upper part of the chamber. The transported flow of seeds passes into a fluidized state, which increases the uniformity of the distribution of seeds through the nozzles 15 .

Use of the proposed pneumatic seeder with a central dosing system in comparison with the known pneumatic seeders with a central dosing system will allow to increase the uniformity of the flow of seeds in coulters and as a result to raise the uniformity of their distribution on the field surface, to increase the throughput capacity of the central dosing system of pneumatic seeders, to reduce the complexity of operation of pneumatic seeders in the seeding process.

\section{References}

1. Rotary disk working bodies as a basic element in the combined units for tillage and seeding / E. I. Trubilin,

K. A. Sokht,

V. I. Konovalov, O. V. Danyukova // Polythematic network electronic scientific journal of Kuban State 
Agrarian University- Krasnodar: Printing house «KubSAU». - 2013. № 91. p. 465497.

2. Isaev Yu. M and Semashkin N. M, Critical Frequency of Rotation in the Spiral Screw Device, International Journal of Mechanical Engineering and Technology, 9(5), 2018, pp. 541-547.

3. Vineet Kumar Bhagat, Anil Kumar Prasad and Arvind Kumar Lai Srivastava, Physical and Mechanical Performance of Luffa-Coir Fiber Reinforced Epoxy Resin Based Hybrid Composites. International Journal of Civil Engineering and Technology, 8(6), 2017, pp. 722-731.

4. A. Jamadin, Z. Ibrahim, N. Mohd Amin, A. Alisibramulisi, N.H. Suliman and M.H.S. Malai Hussin, Dynamic Behaviour of Existing Steel Pedestrian Bridgeintemational Journal of Civil Engineering and Technology, 9(7), 2018, pp. 1443-1450.

5. P. M. Konkov, Mechanization of grain unloading (Moscow, Kolos, 1972).

6. G. O. Harries, Application of a radioizotope to the determination of the annular thicknesl of grain in an anger conveyor. Journal of Agricultural Engineering Research, $1,1962$.

7. Bogus A.E., Grachev E.A. A study of a shock pulse of a roller rib of the planetary threshing device on grain mass // Polythematic network electronic scientific journal of Kuban State Agrarian University- Krasnodar: Printing house «KubSAU». 2018. № 135., p. 188-199.

8. L.D. Landau, E.M. Livshits, Theoretical Physics (Textbook. In 10 vols, T. VI. Hydrodynamics. -4 th ed., Sr. Moscow, 1988, p.736).

9. Papusha S.K., Belousov S.V., Bogus A.E., Konovalov V.I. Theoretical studies of tobacco stalk interaction with a leaf-separating unit // International Journal of Applied Engineering Research. 2016. V. 11. № 8. p. 5610-5613. 